\title{
Forme fruste de fente labio-palatine : présentation d'un cas clinique
}

\author{
Manon Devisse ${ }^{1,}{ }^{*}$, Aurélie Hacquard ${ }^{1}$, Julie Lelièvre ${ }^{2}$, Cédric Lansonneur ${ }^{2}$, \\ Yves Gauvin ${ }^{3}$, Sylvie Boisramé-Gastrin ${ }^{2}$ \\ 1 UFR Odontologie, Service d'Odontologie, CHRU, Brest, France \\ 2 UFR Service d'Odontologie, UF de Chirurgie orale, CHRU, Brest, France \\ ${ }^{3}$ UFR Service d'Oto-rhino-laryngologie, CHRU, Brest, France
}

(Reçu le 27 juin 2012, le 25 juillet 2012)

Mots clés :

fente labio-palatine /

forme mineure/ abortive/fruste / anomalies dentaires / luette bifide
Key words: cleft lip and palate / minor/abortive/ rudimentary form / tooth abnormalities / bifid uvula
Résumé - Les fentes labio-palatines sont les dysmorphoses buccales congénitales les plus fréquentes (1/600 naissances). Elles peuvent être associées à des syndromes polymalformatifs. Malgré une étiologie inconnue, l'hérédité serait un facteur prédominant et il existerait des facteurs prédisposants comme la toxicomanie maternelle, l'alcoolisme parental, la prise de certains médicaments pendant la grossesse. Ces fentes résultent d'une absence ou $d^{\prime}$ une insuffisance de fusion des différents bourgeons faciaux se déroulant de la $5^{\mathrm{e}}$ à la $7^{\mathrm{e}}$ semaine de vie intra-utérine, aboutissant à diverses formes de fentes plus ou moins marquées: les fentes labiales, labio-alvéolaires, palatovélaires, unilatérales ou bilatérales, complètes ou incomplètes. Les fentes labio-palatines de forme fruste, également appelées mineures ou abortives, n'ont pas d'incidence sur l'esthétique : moins visibles, elles sont moins connues. Elles se caractérisent par une luette bifide mais elles peuvent également comporter des anomalies dentaires comme par exemple des dents gémellées ; la dent la plus concernée par ces anomalies étant l'incisive latérale. Dans les formes mineures, il existe également la fente palatine sous-muqueuse qui est une sous-catégorie de fente palatine pour laquelle la plupart des individus atteints ne décrivent aucun symptôme. Elle touche le palais mou et peut entrainer une insuffisance vélo-pharyngée qui affecte la qualité et l'intelligibilité de l'élocution.

Le cas rapporté évoque ces formes frustes. L'odontologiste a un rôle dans la prise en charge de ces patients d'une part pour établir un diagnostic précoce et d'autre part pour réaliser un traitement approprié permettant d'éviter des malpositions, des interférences occlusales, des rétentions provoquant des complications infectieuses. L'approche de ce type de fentes est multidisciplinaire associant chirurgien-dentiste, médecin ORL, orthodontiste.

\begin{abstract}
Minor form of clef lip and palat: a clinical case report. The cleft lip and palate are congenital oral dysmorphoses most common (1/600 births). They can be associated with multiple malformation syndromes. Despite an unknown etiology, heredity is a dominant factor and there would be predisposing factors such as maternal drug use, parental alcoholism, medications during pregnancy. It result from a lack or insufficiency of fusion of different facial buds unfolding of the 5th to the 7th week of intrauterine life, leading to various forms of more or less marked slots: cleft lip, lip and alveolar, palato-velar, unilateral or bilateral, complete or incomplete. The cleft lip and palate in crude form, also called minor or abortive forms, are having no impact on aesthetics: less visible, they are less known. They are characterized by a bifid uvula and also have consequences for dental example geminated teeth, the teeth most affected by the anomalies being the lateral incisor along the slit. In mild forms, there is also the submucosal cleft palate is a subcategory of cleft palate for which most affected individuals do not describe any symptoms. They touches the soft palate and may result in velopharyngeal insufficiency that affects the quality and intelligibility of speech.

The case report is about this minor form. The dentist has a role in the managment of these patients on the one hand for early diagnosis and also to achieve an appropriate treatment to avoid malposition, occlusal interference, causing retention of infectious complications. The dentist also works closely with the orthodontist who will intervene if necessary for alignment of permanent teeth.
\end{abstract}

\footnotetext{
*Correspondance : manon.dev@hotmail.fr
} 


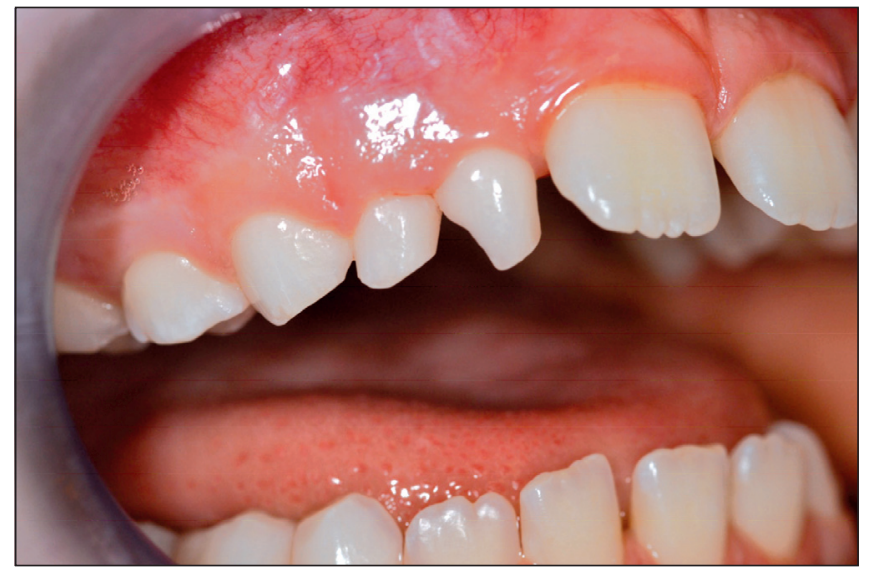

Fig. 1. Duplication de l'incisive latérale temporaire supérieure droite ; elles ont une forme conoïde.

Fig. 1. Duplication of right superior deciduous lateral incisor; they have a conical form.

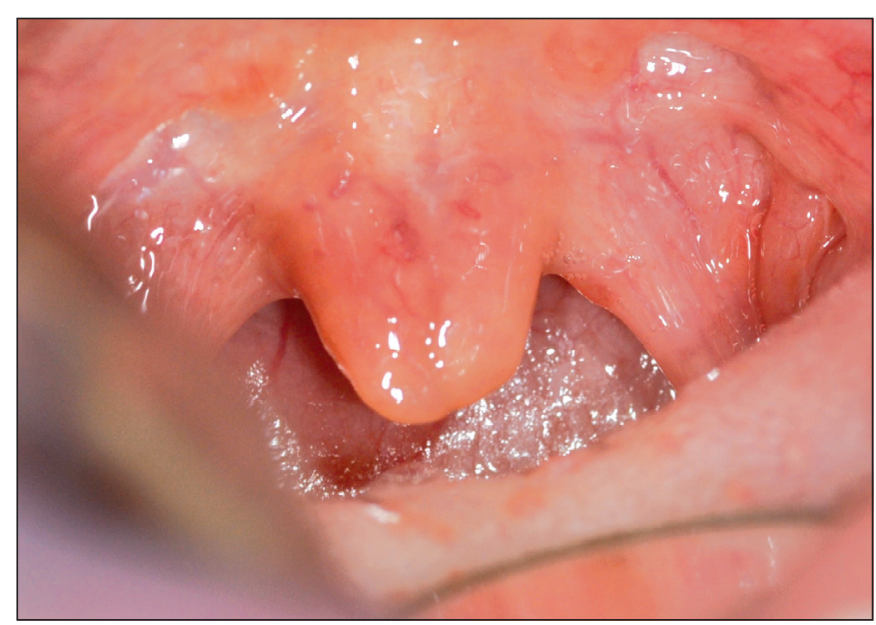

Fig. 2. Bifidité partielle de la luette: les deux extrémités indépendantes de la luette sont réunies uniquement par la muqueuse. Fig. 2. Partial bifid uvula: the two ends of the uvula are met only by mucosa.

Les fentes labio-palatines sont les dysmorphoses buccales congénitales les plus fréquentes (1/600 naissances) et les mieux connues du public $[1,2]$. Elles résultent d'une absence ou d'une insuffisance de la fusion entre les différents bourgeons faciaux qui se déroule de la $5^{\mathrm{e}}$ à la $7^{\mathrm{e}}$ semaine de vie intra-utérine, et qui aboutit à diverses formes de fentes plus ou moins marquées [3, 4]. Cependant, il existe des formes frustes, également appelées mineures ou abortives, qui sont des formes n'ayant pas d'incidence sur l'esthétique : moins visibles, elles sont moins connues. Elles se caractérisent par une luette bifide mais elles peuvent également comporter des

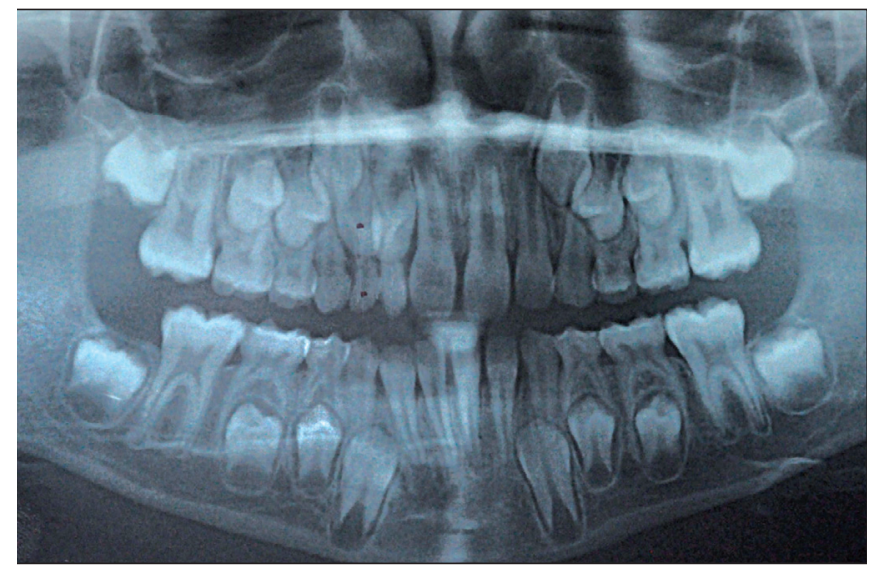

Fig. 3. Orthopantomogramme du cas clinique rapporté. Fig. 3. Orthopantomogram of case report.

anomalies dentaires $[5,6]$. Ces fentes frustes nécessitent ainsi une prise en charge transdisciplinaire alliant l'oto-rhino-laryngologiste, l'odontologiste, l'orthodontiste et l'orthophoniste. Elles ne doivent pas rester méconnues de l'odontologiste afin de permettre une prise en charge adaptée.

Un cas évoquant ces formes frustes est rapporté : il s'agit d'un garçon de 9 ans adressé pour la persistance unilatérale de l'incisive latérale temporaire supérieure droite. Cet article rapporte la prise en charge de ce patient aussi bien pour le diagnostic que pour le plan de traitement. Bien que leur impact soit moins important que celui des formes majeures de fentes, ces formes abortives ne doivent pas rester ignorées.

\section{Observation}

Un jeune patient, âgé de 9 ans, est adressé dans le service d'0dontologie du Centre hospitalier régional universitaire de Brest pour la persistance de l'incisive latérale temporaire supérieure droite.

L'examen clinique permet de retrouver une duplication de cette dent (52 et 52 bis) (Fig. 1). Cette anomalie ne crée pas de malocclusion ou de dysharmonie dento-maxillaire grâce aux diastèmes physiologiques présents en dentition temporaire. En outre, il est observé une bifidité de la luette (Fig. 2) qui n'engendre pas de problèmes d'élocution. L'orthopantomogramme (Fig. 3) objective la rétention de l'incisive latérale permanente elle-même gémellée (12 et 12bis) et de la canine permanente. Le manque de place causé par la présence d'un germe supplémentaire (12bis) engendre le retard d'éruption de la canine définitive. Cette anomalie de nombre par excès n'est pas associée à une anomalie de forme : en effet, l'anatomie 
coronaire et radiculaire des incisives latérales temporaires et définitives reste respectée donnant des dents supplémentaires eumorphiques.

Le plan de traitement consiste à corriger cette anomalie de nombre en réalisant l'avulsion des incisives latérales temporaires qui ont un retard de chute et d'une des deux incisives latérales permanentes ce qui devrait permettre l'évolution avec un chemin d'éruption normal de la canine permanente.

Sur une radiographie rétro-alvéolaire, on a réalisé la mesure de la largeur mésio-distale afin de prévoir une éventuelle dysharmonie dento-maxillaire et de choisir la thérapeutique la mieux adaptée. En effet, grâce à ces mesures et selon la position des germes de la canine et des deux incisives gémellées (12 et 12bis), il est préférable de réaliser l'avulsion de l'incisive latérale définitive bis. (Fig. 4). Un avis orthodontique sera demandé par la suite.

\section{Commentaires}

Les fentes labio-palatines entrent dans le cadre des neurocristopathies par défaut dans la migration et dans le rôle de différenciation et d'induction des cellules de la crête neurale embryonnaire. La fente est créée par la persistance de l'écart entre les bourgeons maxillaires, par absence ou insuffisance de fusion.

Dans $70 \%$ des cas, cette malformation est une anomalie isolée et, dans $30 \%$ des cas, elle est associée à un syndrome (environ 300 syndromes comportent une fente labio-palatine) (Tab. I) [7-9]. En effet, l'anomalie détectable à partir de la $16^{\mathrm{e}}$ semaine de vie intra-utérine à l'échographie nécessite une recherche de pathologie associée, comme par exemple le syndrome de Pierre Robin qui regroupe une hypoplasie congénitale de la mandibule, une glossoptose et une fente palatine.

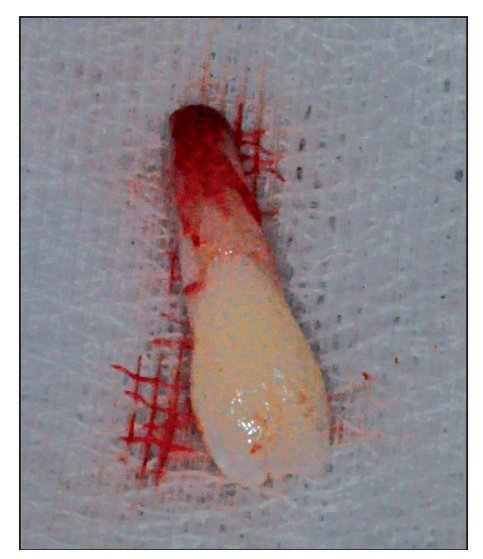

Fig. 4. Dent définitive surnuméraire eumorphique (12bis). Fig .4. Supplemental supernumerary permanent tooth (12bis).

Il existe différentes formes de fente :

- La fente labiale concerne la lèvre supérieure allant parfois jusqu'au seuil narinaire, l'aile du nez est alors étalée.

- La fente labio-alvéolaire intéresse la lèvre supérieure avec une fissure de la région alvéolaire, voire de tout le palais primaire. La fente se situe habituellement dans la zone du germe de l'incisive latérale.

- La fente vélaire touche le voile du palais avec une étendue plus ou moins marquée du rebord postérieur du palais osseux.

- La fente vélo-palatine intéresse le voile et le palais osseux jusqu'au foramen incisif et expose le vomer.

La fente labio-palatine ou labio-maxillo-palatine constitue la forme totale, touchant le palais primaire et le palais secondaire. Des formes frustes ou abortives sont également décrites comme la fente sous-muqueuse du voile qui se manifeste par

Tableau I. Association de fentes aux syndromes les plus rencontrés.

Table I. Association between oral clefts and common congenital anomalies.

\begin{tabular}{|c|c|c|}
\hline Pathologie & Type de fente & Symptôme associés \\
\hline Syndrome de Pierre Robin & Fente palatine & $\begin{array}{l}\text { Hypoplasie congénitale de la mandibule } \\
\text { Glossoptose }\end{array}$ \\
\hline Syndrome de Hay-Wells & Fente palatine ou labiale & $\begin{array}{l}\text { Dysplasie ectodermique } \\
\text { Ankyloblépharon filiforme }\end{array}$ \\
\hline Syndrome de Baraitser & Fente labio-palatine & $\begin{array}{l}\text { Craniosynostose } \\
\text { Retard mental }\end{array}$ \\
\hline Syndrome de Rosselli-Gulienetti & Fente labio-palatine & $\begin{array}{l}\text { Ectodermie } \\
\text { Retard mental } \\
\text { Syndactylie aux mains et aux pieds }\end{array}$ \\
\hline Syndrome de van der Woude & Fente labiale et/ou palatine & Fistule de la lèvre inférieure \\
\hline
\end{tabular}




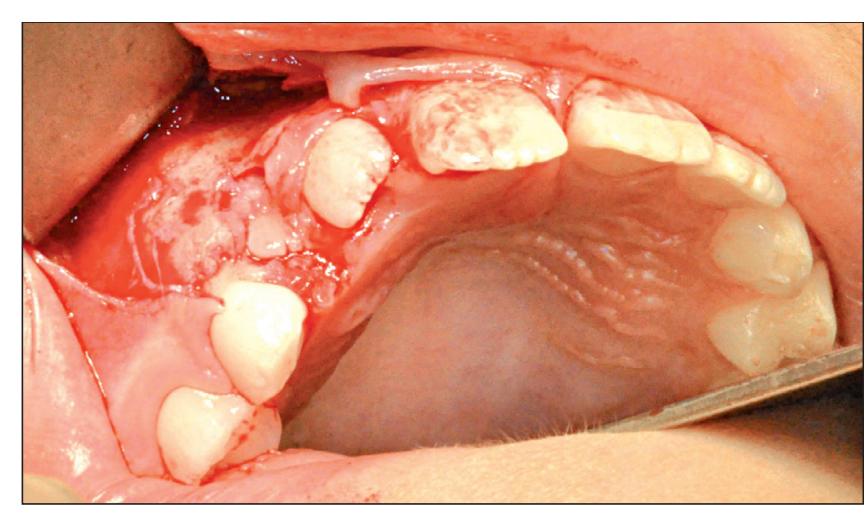

Fig. 5. Mise en évidence des germes 12 et 12 bis après levée d'un lambeau de pleine épaisseur.

Fig. 5. Visualization of germs 12 and 12 bis after full-thickness flap élévation.

un défaut de soudure des berges musculaires. Elle n'est souvent décelée que par la bifidité de la luette et la transparence anormale du voile à ce niveau [10].

Les fentes peuvent être uni ou bilatérales, symétriques ou asymétriques. Elles peuvent également être mineures comme le montre le cas clinique décrit. Ces formes mineures ou frustes sont d'une incidence faible, mais ceci peut se justifier par le fait qu'elles sont peu connues des chirurgiens-dentistes, et peuvent ainsi passer inaperçues. Cliniquement, le principal signe évoqué concerne l'incisive latérale [11, 12]. En fait, celle-ci étant située dans la zone de division alvéolaire, elle peut être soit dupliquée soit absente, créant une anomalie de nombre respectivement par excès ou par défaut. La denture temporaire et/ou la denture définitive peuvent être atteintes, les germes dupliqués présenter une anomalie de forme (forme conique) ou être eumorphiques. Un traitement orthodontique est souvent nécessaire [13]. L'autre signe clinique concerne la bifidité de la luette : elle est totale ou partielle. Chez certains patients, cette anomalie entraine des difficultés d'élocution et peut nécessiter la prise en charge par un orthophoniste.

Conflits d'intérêt : aucun

\section{Références}

1. Kouskoura T, Fragou N, Alexiou M, John N, Sommer L, Graf D, Katsaros C, Mitsiadis TA. The genetic basis of craniofacial and dental abnormalities. Schweiz Monatsschr Zahnmed 2011; 121:636-46.

2. Mars M, Sell D, Habel A. Management of cleft lip and palate in the developing world. John Wiley, New York, 2008.

3. Mossey PA, Little J, Munger RG, Dixon MJ, Shaw WC. Cleft lip and palate. Lancet 2009;374:1773-85.

4. Chatzistavrou E, Bruce Ross R, Tompson B-D, Johnston M-C. Predisposing factors to formation of cleft lip and palate: inherited craniofacial skeletal morphology. Cleft Palate Craniofac J 2004;41:613-21.

5. Yuzuriha S, Mulliken J-B. Minor-form, microform, and minimicroform cleft lip: anatomical features, operative techniques, and revisions. Plast Reconstr Surg 2008 122:1485-93.

6. Thaller S-R, Lee T-J. Microform cleft lip associated with a complete cleft palate. Cleft Palate Craniofac J 1995;32:247-50.

7. Lehman J-A, Fishman JR-A, Neiman G-S. Treatment of cleft palate associated with Robin sequence: appraisal of risk factors. Cleft Palate Craniofac J 1995;32:25-9.

8. Sekhon P-S, Ethunandan M, Markus A-F, Krishman G, Bhasker Rao C. Congenital anomalies associated with cleft lip and palate: an analysis of 1623 consecutive patients. Cleft Palate Craniofac J 2011;48:371-8.

9. Shivaprakash PK, Joshi HV, Noorani H, Reddy V. Ectrodactyly, ectodermal dysplasia, and cleft lip/palate syndrome: a case report of "incomplete syndrome". Contemp Clin Den 2012;3:115-7.

10. Nasser M, Fedorowicz Z, Newton JT, Nouri M. Interventions for the management of submucous cleft palate, Cochrane Database Syst Rev 2008;23:CD006703.

11. Rakotoarison R-A, Rakotoarivony A-E, Ralaiarimanana F-L, Andriambololo-Nivo R, Féki A. Étude des anomalies dentaires associées aux fentes labiopalatines : à propos d'une série de 85 cas. Med Buccale Chir Buccale 2011;17:7-14.

12. Ruiz L, Maya R, D'Alpino P, Atta M, Svizero N. Prevalence of enamel defects in permanent teeth of patients with complete lip and palate, Cleft Palate Craniofac J 2012;31 [Epub ahead of print].

13. Freitas JA, Garib DG, Oliveira M, Lauris Rde C, Almeida AL, Neves LT, Trindade-Suedam IK, Yaedú RY, Soares S, Pinto JH. Rehabilitative treatment of cleft lip and palate: experience of the Hospital for rehabilitation of craniofacial anomalies - USP (HRACUSP)- Part 2: Pediatric dentistry and orthodontics. J Appl Oral Sci 2012;20:272-85.

14. Vogels WE, Aartman IH, Veerkamp JS. Dental fear in children with a cleft lip and/or palate, Cleft Palate Craniofac J 2011;48:736-40.

15. Cooney PV. Analyse de l'environnement des cliniques de traitement de la fente labiale et de la fente palatine et des programmes de soins dentaires au Canada. 2010.

16. Hall M, Gibson BJ, James A, Rodd H. Children's and adolescent's perspectives on cleft lip and/or palate. Cleft Palate Craniofac J 2012;13 [Epub ahead of print]. 